VOL. 61 (2000) [177-187]

\title{
ON THE ARC INDEX OF AN ADEQUATE LINK
}

\author{
Chan-Young Park and Myoungsoo Seo
}

In 1996, Cromwell and Nutt conjectured that $\alpha(L)-c(L)=2$ for a link $L$ if and only if $L$ is alternating. In this paper we calculate that $\alpha(L)=c(L)$ for some non-alternating pretzel links $L$, define a new invariant $\rho(L)$ of adequate links $L$ and show that for each non-negative integer $n$, there is a prime adequate knot $K$ such that $\alpha(K)-c(K)=-2 n$. We conjecture that $\alpha(L)-c(L)=2 \rho(L)$ for any adequate link $L$.

\section{INTRODUCTION}

An open book decomposition of the 3 -sphere $\mathbf{S}^{3}$ consists of open discs as pages and an unknotted circle as a binding circle. Every link $L$ can be embedded in an open book with finitely many pages so that it meets each page in a simple arc. Such an embedding is called an arc presentation of $L$. The minimum number of pages required to present a given link $L$ in this manner is a knot invariant and it is called the arc index of $L$ which is denoted by $\alpha(L)$.

In 1994, the notion of an arc index was introduced by Birman and Menasco [3] in their study of the braid index of satellite links. In 1995, Cromwell [4] gave basic properties on the arc index of links and in 1996, Cromwell and Nutt [6] introduced a method to construct binding circles on some link diagrams and found bounds on the arc index and conjectured that $\alpha(L) \leqslant c(L)+2$ for a link $L$ and that equality holds if and only if $L$ is alternating, where $c(L)$ is the crossing number. Recently, this conjecture was proved affirmatively by Bae and Park [1]. In 1998, Morton and Beltrami [9] showed that $\alpha(L) \geqslant$ breadth $_{a} F_{L}(a, z)+2$, using the Dubrovnik framed version of the Kauffman polynomial and the notion of a stacked tangle, where $F_{L}(a, z)$ is the Kauffman polynomial of $L$ and breadth $F_{L}(a, z)$ is the $a$-spread of the polynomial $F_{L}(a, z)$. In 1997, Beltrami and Cromwell [2] proved that for a semi-alternating link $L$, breadth $_{a} F_{L}(a, z) \geqslant c(L)-2$

\section{Received December 21, 1999}

This work was partially supported by the Korea Research Foundation made in the program year of 1998 and by TGRC-KOSEF.

Copyright Clearance Centre, Inc. Serial-fee code: 0004-9727/00 \$A2.00+0.00. 
and in 1998 Cromwell [5] showed that for an alternating link $L$, breadth $F_{L}(a, z) \geqslant c(L)$, using the Kauffman polynomial of an adequate link.

In this paper we calculate that $\alpha(L)=c(L)$ for some non-alternating pretzel links $L$, define a new invariant of adequate links and show that for each non-negative integer $n$, there is a prime adequate knot $K$ such that $\alpha(K)-c(K)=-2 n$.

Unless otherwise stated, all links considered in this paper are assumed to be nonsplit. Note that all diagrams of non-split links are connected.

\section{AN INVARIANT OF ADEQUATE LINKS}

In this section, we define an invariant $\rho(L)$ of an adequate link $L$.

We start by introducing some notations. Let $D$ be a connected link diagram in a plane. Then the diagram $D$ divides the plane into several regions. Starting with the unbounded region, we can colour the regions either black or white so that neighbouring regions never have the same colour. We colour the unbounded region white. Let $G$ be the connected planar graph associated with the diagram $D$ in which the vertices of $G$ correspond to the black regions of $D$ and the edges of $G$ correspond to the crossings of $D$. Assign a sign to each edge of $G$ as depicted in Figure 2.1 so that the graph $G$ is completely determined by the diagram $D$.
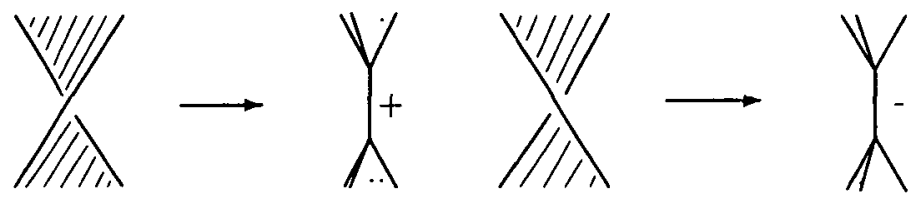

Figure 2.1

Given a graph $G$ associated with a connected link diagram $D$, let $G_{+}$be the subgraph of $G$ consisting of all vertices of $G$ and the positive edges of $G$ and let $\overline{G_{+}}$be the quotient graph obtained from $G_{+}$by identifying those pairs of vertices which are ends of a path consisting of negative edges of $G$. The graphs $G_{-}$and $\overline{G_{-}}$are defined likewise. For any graph $G, p_{0}(G)$ denotes the number of components of $G$ and $p_{1}(G)$ denotes the least number of edges of $G$ whose deletion destroys all the circuits of $G$. Notice that $p_{0}(G)$ and $p_{1}(G)$ are the ranks of homology groups $H_{0}(G)$ and $H_{1}(G)$ respectively.

For a diagram $D$ of an oriented link $L$ with writhe $w(D)$, the regular isotopy invariant $\Lambda_{D}(a, z)$ is defined by $\Lambda_{D}(a, z)=a^{w(D)} F_{L}(a, z)$, where $F_{L}(a, z)$ is the Kauffman polynomial of $L$. Alternatively, the polynomial $\Lambda_{D}(a, z)$ can be defined by the following four axioms: 
(1) $\Lambda_{D}(a, z)$ is a regular isotopy invariant of diagrams of $L$,

(2) $\Lambda_{D}=1$ if $D$ is a simple closed curve,

(3) $\Lambda_{D_{1}}=a \Lambda_{D_{3}}$ and $\Lambda_{D_{2}}=a^{-1} \Lambda_{D_{3}}$, where $D_{1}, D_{2}$ and $D_{3}$ are the same diagrams except within the neighbourhoods depicted as in Figure 2.2, and

(4) $\Lambda_{D_{+}}+\Lambda_{D_{-}}=z\left(\Lambda_{D_{0}}+\Lambda_{D_{\infty}}\right)$ where $D_{+}, D_{-}, D_{0}$ and $D_{\infty}$ are the same except within the neighbourhoods depicted as in Figure 2.3.

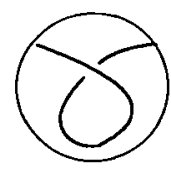

$D_{1}$

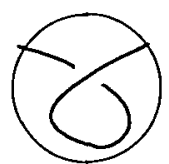

$D_{2}$

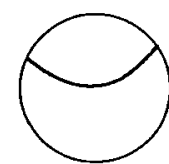

$D_{3}$

Figure 2.2

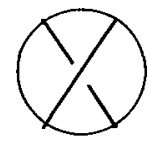

$D_{+}$

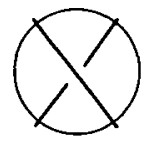

D.

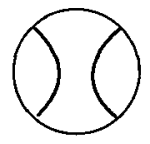

$D_{O}$

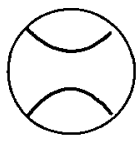

$D_{\infty}$

Figure 2.3

Write $\Lambda_{D}(a, z)=\sum u_{r s} a^{r} z^{s}$ and let $c(D)=n$ where $c(D)$ denotes the crossing number of the link diagram $D$. Then $r+s \leqslant n$ and $-r+s \leqslant n$ if $u_{r s}$ is nonzero [11]. Put $\phi_{D}^{+}(t)=\sum_{i} u_{i, n-i} t^{i}$ and $\phi_{D}^{-}(t)=\sum_{i} u_{-i, n-i} t^{i}$. Then there are no negative powers of $t$ occurring in $\phi_{D}^{+}(t)$ or $\phi_{D}^{-}(t)$. In 1988, Thistlethwaite showed the following proposition by using a relation between the regular isotopy invariant $\Lambda_{D}(a, z)$ of a link diagram $D$ and the Tutte polynomial of the associated graph $G$ of $D$.

Proposition 2.1. [12] Let $D$ be a connected link diagram with $c(D) \geqslant 1$. Then the following hold.

(1) $\phi_{D}^{+}(t) \neq 0$ if and only if $G_{+}$has no isthmus and $\overline{G_{-}}$has no loop.

(2) $\phi_{\bar{D}}^{-}(t) \neq 0$ if and only if $G_{-}$has no isthmus and $\overline{G_{+}}$has no loop.

(3) If $\phi_{D}^{+}(t) \neq 0$, then max $\operatorname{deg} \phi_{D}^{+}(t)=p_{1}\left(G_{+}\right)+\left|V\left(\overline{G_{-}}\right)\right|-1$.

(4) If $\phi_{D}^{-}(t) \neq 0$, then max deg $\phi_{D}^{-}(t)=p_{1}\left(G_{-}\right)+\left|V\left(\overline{G_{+}}\right)\right|-1$.

(5) $\phi_{D}^{+}(t) \neq 0$ and $\phi_{D}^{-}(t) \neq 0$ if and only if $D$ is adequate.

Here $|S|$ denotes the cardinality of a set $S$.

A link diagram $D$ is said to be + adequate if $D$ has at least one crossing, and for each crossing in the Kauffman state $s_{+} D$ formed by nullifying each crossing according to rule $\mathrm{P}$ 
in Figure 2.4, the two segments of the image part in rule $\mathrm{P}$ belong to different components of $s_{+} D$. If $D$ satisfies the corresponding condition for the state $s_{-} D$ obtained by nullifying each crossing according to rule $\mathrm{N}$ in Figure 2.4 , then $D$ is said to be - adequate. A link diagram $D$ is said to be adequate if it is both + adequate and - adequate and a link is called an adequate link if it has an adequate diagram.

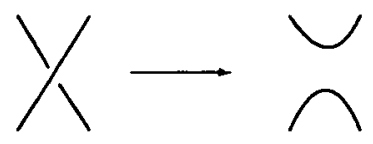

The rule $\mathrm{N}$

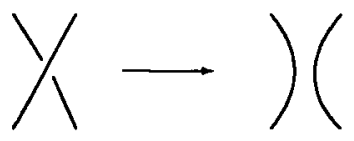

The rule $\mathrm{P}$

Figure 2.4

For a directed graph $G$, the subgraphs $G_{+}$and $G_{-}$intersect only in vertices of $G$. Thus $\left|G_{+} \cap G_{-}\right|$is the number of vertices of $G_{+} \cap G_{-}$.

LEMMA 2.2. Let $D$ be a connected adequate diagram of an adequate link $L$ and let $G$ be the associated graph of $D$. Then the following hold.

(1) $p_{1}\left(G_{+}\right)=p_{0}\left(G_{+}\right)+\left|E\left(G_{+}\right)\right|-\left|V\left(G_{+}\right)\right|$and $p_{1}\left(G_{-}\right)=p_{0}\left(G_{-}\right)+\left|E\left(G_{-}\right)\right|-\left|V\left(G_{-}\right)\right|$.

(2) $\left|V\left(\overline{G_{+}}\right)\right|=\left|V\left(G_{+}\right)\right|-\left|G_{+} \cap G_{-}\right|+p_{0}\left(G_{-}\right)$and $\left|V\left(\overline{G_{-}}\right)\right|=\left|V\left(G_{-}\right)\right|-\left|G_{+} \cap G_{-}\right|+p_{0}\left(G_{+}\right)$.

(3) $\max \operatorname{deg} \phi_{D}^{+}(t)+\max \operatorname{deg} \phi_{D}^{-}(t)=\left|E\left(G_{+}\right)\right|+\left|E\left(G_{-}\right)\right|+2\left(p_{0}\left(G_{+}\right)+p_{0}\left(G_{-}\right)-\right.$ $\left.\left|G_{+} \cap G_{-}\right|\right)-2$.

Proof: (1) Assume that $G_{+}$has $n$ components $G_{1}, G_{2}, \ldots, G_{n}$. Then $p_{0}\left(G_{+}\right)=n$. Since each $G_{i}$ is planar, $p_{1}\left(G_{i}\right)$ equals the number of bounded regions of $G_{i}$. Thus $p_{1}\left(G_{i}\right)=\left|E\left(G_{i}\right)\right|-\left|V\left(G_{i}\right)\right|+1$. Since $\left|E\left(G_{+}\right)\right|=\sum_{i=1}^{n}\left|E\left(G_{i}\right)\right|$ and $\left|V\left(G_{+}\right)\right|=\sum_{i=1}^{n}\left|V\left(G_{i}\right)\right|$, $p_{1}\left(G_{+}\right)=\sum_{i=1}^{n} p_{1}\left(G_{i}\right)=\sum_{i=1}^{n}\left|E\left(G_{i}\right)\right|-\sum_{i=1}^{n}\left|V\left(G_{i}\right)\right|+n=p_{0}\left(G_{+}\right)+\left|E\left(G_{+}\right)\right|-\left|V\left(G_{+}\right)\right|$. Similarly, $p_{1}\left(G_{-}\right)=p_{0}\left(G_{-}\right)+\left|E\left(G_{-}\right)\right|-\left|V\left(G_{-}\right)\right|$.

(2) Note that $\overline{G_{+}}$is the quotient graph obtained from $G_{+}$by identifying those pairs of vertices which are ends of a path consisting of negative edges of $G$, while $G_{+} \cap G_{-}$is a vertex graph and the vertices of $G_{+} \cap G_{-}$are ends of a path consisting of negative edges of $G$. Since the number of identified vertices in $\overline{G_{+}}$is $p_{0}\left(G_{-}\right),\left|V\left(\overline{G_{+}}\right)\right|=\left|V\left(G_{+}\right)\right|-$ $\left|G_{+} \cap G_{-}\right|+p_{0}\left(G_{-}\right)$. Similarly, $\left|V\left(\overline{G_{-}}\right)\right|=\left|V\left(G_{-}\right)\right|-\left|G_{+} \cap G_{-}\right|+p_{0}\left(G_{+}\right)$.

(3) The equality follows from (1), (2) and [12].

Notice that there is a one-to-one correspondence between the crossings of $L$ and the edges of $G$, which implies $c(D)=\left|E\left(G_{+}\right)\right|+\left|E\left(G_{-}\right)\right|$. 
Now we put $\rho(D)=p_{0}\left(G_{+}\right)+p_{0}\left(G_{-}\right)-\left|G_{+} \cap G_{-}\right|$for a connected diagram $D$ of a link $L$. Then we get the following

THEOREM 2.3. Let $D$ be an adequate diagram of an adequate link $L$ and let $G$ be the associated graph of $D$. Then

$$
\text { breadth }_{a} \Lambda_{D}(a, z) \geqslant c(D)+2 \rho(D)-2 .
$$

Proof: Since

$\max \operatorname{deg}_{a} \Lambda_{D}(a, z) \geqslant \max \operatorname{deg} \phi_{D}^{+}(t)$ and $\min _{\operatorname{deg}_{a}} \Lambda_{D}(a, z) \leqslant-\max \operatorname{deg} \phi_{D}^{-}(t)$, from Lemma 2.2 we have

$$
\begin{aligned}
\text { breadth }_{a} \Lambda_{D}(a, z)= & \max \operatorname{deg}_{a} \Lambda_{D}(a, z)-\min \operatorname{deg}_{a} \Lambda_{D}(a, z) \\
\geqslant & \max \operatorname{deg} \phi_{D}^{+}(t)+\max \operatorname{deg} \phi_{D}^{-}(t) \\
= & \left|E\left(G_{+}\right)\right|+\left|E\left(G_{-}\right)\right| \\
& +2\left(p_{0}\left(G_{+}\right)+p_{0}\left(G_{-}\right)-\left|G_{+} \cap G_{-}\right|\right)-2 \\
= & c(D)+2 \rho(D)-2 .
\end{aligned}
$$

Now for an adequate link $L$, we define $\rho(L)=\rho(D)=p_{0}\left(G_{+}\right)+p_{0}\left(G_{-}\right)-\left|G_{+} \cap G_{-}\right|$ for some adequate diagram $D$ of $L$.

THEOREM 2.4. $\rho(L)$ is an invariant of an adequate link $L$.

Proof: Let $L$ be an adequate link and let $D_{1}, D_{2}$ be adequate diagrams of $L$ with writhes $w\left(D_{1}\right)$ and $w\left(D_{2}\right)$, respectively. Then $w\left(D_{1}\right)=w\left(D_{2}\right)$ [12]. Since $\Lambda_{D_{1}}(a, z)=a^{w\left(D_{1}\right)} F_{L}(a, z)=a^{w\left(D_{2}\right)} F_{L}(a, z)=\Lambda_{D_{2}}(a, z), \phi_{D_{1}}^{+}(t)=\phi_{D_{2}}^{+}(t)$ and $\phi_{D_{1}}^{-}(t)=$ $\phi_{D_{2}}^{-}(t)$. Thus max $\operatorname{deg} \phi_{D_{1}}^{+}(t)+\max \operatorname{deg} \phi_{D_{1}}^{-}(t)=\max \operatorname{deg} \phi_{D_{2}}^{+}(t)+\max \operatorname{deg} \phi_{D_{2}}^{-}(t)$. Since $\max \operatorname{deg} \phi_{D_{1}}^{+}(t)+\max \operatorname{deg} \phi_{D_{1}}(t)=c\left(D_{1}\right)+2 \rho\left(D_{1}\right)-2$, in the proof of Theorem 2.3, and $c\left(D_{1}\right)=c\left(D_{2}\right)$ [12], we have $\rho\left(D_{1}\right)=\rho\left(D_{2}\right)$. Thus $\rho(L)$ is well-defined and $\rho(L)$ is a link invariant for adequate links.

Since breadth ${ }_{a} \Lambda_{D}(a, z)=\operatorname{breadth}_{a} F_{D}(a, z)$ and $\alpha(L) \geqslant \operatorname{breadth}_{a} F_{D}(a, z)+2$, we have the following

CoRollary 2.5. Let $L$ be an adequate link. Then breadth $F_{L}(a, z) \geqslant c(L)+$ $2 \rho(L)-2$ and hence $\alpha(L)-c(L) \geqslant 2 \rho(L)$.

Corollary 2.6. Let $L$ be an alternating link. Then $\rho(L)=1$.

Proof: Let $D$ be an alternating diagram of an alternating link $L$ and let $G$ be the associated graph of $D$. Then $D$ is an adequate diagram. Since $D$ is alternating, the signs of all edges of $G$ are the same. That is, one of the subgraphs $G_{+}$and $G_{-}$is empty. Suppose $G_{+}$is empty. Since $D$ is connected and $G=G_{-}, p_{0}\left(G_{-}\right)=1$ and $p_{0}\left(G_{+}\right)=0$. Hence $\rho(L)=p_{0}\left(G_{+}\right)+p_{0}\left(G_{-}\right)-\left|G_{+} \cap G_{-}\right|=1$. 
In [1], Bae and Park showed that $\alpha(L)-c(L)=2$ for an alternating link $L$. If $L$ is an alternating link, then $L$ is adequate, $\rho(L)=1$ and $\alpha(L)-c(L)=2 \rho(L)$. We conjecture that $\alpha(L)-c(L)=2 \rho(L)$ for any adequate link $L$.

\section{PRETZEL LiNKS WITH ADEQUATE DIAGRAMS}

For non-zero integers $q_{1}, q_{2}, \ldots, q_{m}$, let $P\left(q_{1}, q_{2}, \ldots, q_{m}\right)$, called a pretzel link, be the link with the regular diagram shown in Figure 3.1.

If $\left(q_{1}^{\prime}, q_{2}^{\prime}, \ldots, q_{m}^{\prime}\right)$ is a cyclic permutation of $\left(q_{1}, q_{2}, \ldots, q_{m}\right)$, then $P\left(q_{1}^{\prime}, q_{2}^{\prime}, \ldots, q_{m}^{\prime}\right)$ and $P\left(q_{1}, q_{2}, \ldots, q_{m}\right)$ are equivalent. If $q_{i}= \pm 1$, then $P\left(q_{1}, q_{2}, \ldots, q_{i}, \ldots, q_{m}\right)$ is equivalent to $P\left(q_{i}, q_{1}, q_{2}, \ldots, \widehat{q}_{i}, \ldots, q_{m}\right)$ where $\widehat{q}_{i}$ means the deletion of $q_{i}$. So any pretzel link can be deformed into the form $P\left(\varepsilon, \ldots, \varepsilon, p_{1}, \ldots, p_{n}\right)$ where $\varepsilon= \pm 1$ and $\left|p_{i}\right|>1$. We denote $P\left(\varepsilon, \ldots, \varepsilon, p_{1}, \ldots, p_{n}\right)$ by $P\left(k \varepsilon ; p_{1}, \ldots, p_{n}\right)$ where $k$ is the number of $\varepsilon$ 's. If $k>0$ and $p_{i}=-2 \varepsilon$, then $P\left(k \varepsilon ; p_{1}, \ldots, p_{i}, \ldots, p_{n}\right)$ has the same link type as $P\left((k-1) \varepsilon ; p_{1}, \ldots,-p_{i}, \ldots, p_{n}\right)$. Hence we may assume that none of the $p_{i}$ 's are equal to $-2 \varepsilon$, when $k>0$. Then we have that $P\left(k \varepsilon ; p_{1}, \ldots, p_{n}\right)$ is a knot if and only if either (1) $n \geqslant 0$ and $p_{1}, \ldots, p_{n}, n+k$ are odd or (2) $n \geqslant 1$ and just one of the $p_{i}$ 's is even. We call the knot $P\left(k \varepsilon ; p_{1}, \ldots, p_{n}\right)$ a pretzel knot of odd type in the case of $(1)$ and a pretzel knot of even type in the case of (2).

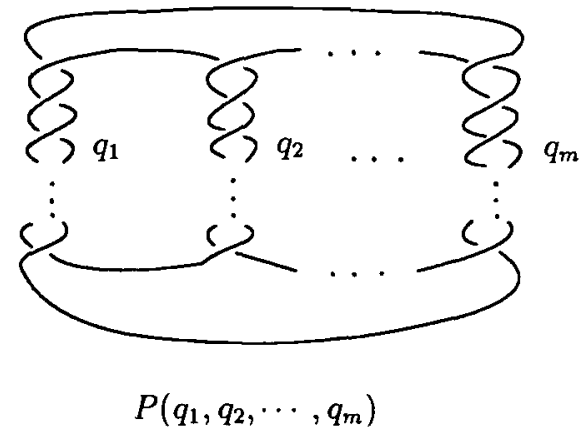

Figure 3.1

We denote $P\left(0 \varepsilon ; p_{1}, \ldots, p_{n}\right)$ by $P\left(p_{i} ; n\right)$. Then $P\left(p_{i} ; n\right)$ is a pretzel link with $\left|p_{i}\right|>1$, for all $i=1, \ldots, n$. Let $N_{+} P\left(p_{i} ; n\right)=\left\{p_{i} \mid p_{i}>0\right\}$ and $N_{-} P\left(p_{i} ; n\right)=\left\{p_{i} \mid p_{i}<0\right\}$ and let $n_{+} P\left(p_{i} ; n\right)$ and $n_{-} P\left(p_{i} ; n\right)$ denote the number of elements of $N_{+} P\left(p_{i} ; n\right)$ and $N_{-} P\left(p_{i} ; n\right)$ respectively. We can easily get the following

Proposition 3.1. Let $P\left(p_{i} ; n\right)$ be a pretzel link. If $n_{+} P\left(p_{i} ; n\right)=0$ or $n_{-} P\left(p_{i} ; n\right)=0$, then we have

(1) $P\left(p_{i} ; n\right)$ is an alternating link and hence is an adequate link, 
(2) $\rho\left(P\left(p_{i} ; n\right)\right)=1$ and

(3) $\quad \alpha\left(P\left(p_{i} ; n\right)\right)=\left|p_{1}\right|+\cdots+\left|p_{n}\right|+2=c\left(P\left(p_{i} ; n\right)\right)+2$.

Now we consider the special class of pretzel links such that $n_{+} P\left(p_{i} ; n\right) \geqslant 2$ and $n_{-} P\left(p_{i} ; n\right) \geqslant 2$. Let $D\left(p_{i} ; n\right)$ denote the regular diagram of $P\left(p_{i} ; n\right)$ in Figure 3.1 . We call the diagram $D\left(p_{i} ; n\right)$ the pretzel diagram of $P\left(p_{i} ; n\right)$.

LEMMA 3.2. Let $D\left(p_{i} ; n\right)$ be the pretzel diagram of a pretzel link $P\left(p_{i} ; n\right)$ and let $G$ be the associated graph of $D\left(p_{i} ; n\right)$. Assume that $n_{+} P\left(p_{i} ; n\right) \geqslant 2$ and $n_{-} P\left(p_{i} ; n\right) \geqslant 2$. Then we have

(1) $G_{+}$and $G_{-}$have no isthmus,

(2) $\overline{G_{+}}$and $\overline{G_{-}}$have no loop and

(3) $\rho\left(D\left(p_{i} ; n\right)\right)=0$.

Proof: Since $n_{+} P\left(p_{i} ; n\right) \geqslant 2$ and $n_{-} P\left(p_{i} ; n\right) \geqslant 2, G_{+}$and $G_{-}$have no isthmus. And since $\left|p_{i}\right| \geqslant 2, i=1, \ldots, n, \overline{G_{+}}$and $\overline{G_{-}}$have no loop. Since $G_{+}$and $G_{-}$are connected and $G_{+} \cap G_{-}$has two vertices, $p_{0}\left(G_{+}\right)=1, p_{0}\left(G_{-}\right)=1$ and $\left|G_{+} \cap G_{-}\right|=2$. Thus $\rho\left(D\left(p_{i} ; n\right)\right)=p_{0}\left(G_{+}\right)+p_{0}\left(G_{-}\right)-\left|G_{+} \cap G_{-}\right|=0$.

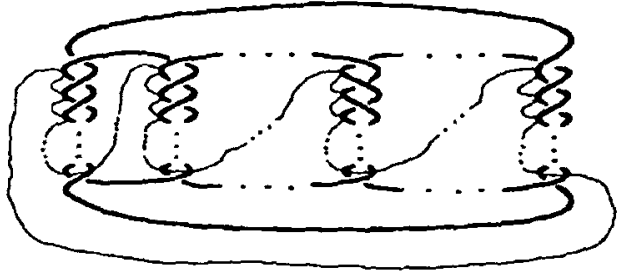

Figure 3.2

TheOREM 3.3. Let $P\left(p_{i} ; n\right)$ be a pretzel link. Assume that $n_{+} P\left(p_{i} ; n\right) \geqslant 2$ and $n_{-} P\left(p_{i} ; n\right) \geqslant 2$. Then we have

(1) $P\left(p_{i} ; n\right)$ is an adequate link,

(2) $\rho\left(P\left(p_{i} ; n\right)\right)=0$ and

(3) $\alpha\left(P\left(p_{i} ; n\right)\right)=c\left(P\left(p_{i} ; n\right)\right)$.

Proof: (1) follows from Proposition 2.1 and Lemma 3.2 and (2) follows from Theorem 2.4 and Lemma 3.2.

(3) Since the graphs $G_{+}$and $G_{-}$are connected, $p_{0}\left(G_{+}\right)=p_{0}\left(G_{-}\right)=1$. Since $G_{+}$ and $G_{-}$intersect in two vertices, $\left|G_{+} \cap G_{-}\right|=2$. By Corollary 2.5, $\alpha\left(P\left(p_{i} ; n\right)\right) \geqslant$ $c\left(P\left(p_{i} ; n\right)\right)+2 p\left(P\left(p_{i} ; n\right)\right)=c\left(P\left(p_{i} ; n\right)\right)$. Since $n_{+} P\left(p_{i} ; n\right) \neq 0$ and $n_{-} P\left(p_{i} ; n\right) \neq 0$, the pretzel diagram $D\left(p_{i} ; n\right)$ of the adequate link $P\left(p_{i} ; n\right)$ has a binding circle which intersects $D\left(p_{i} ; n\right)$ in $c\left(P\left(p_{i} ; n\right)\right)$ points as in Figure 3.2. Hence $\alpha\left(P\left(p_{i} ; n\right)\right)=c\left(P\left(p_{i} ; n\right)\right)$.

For a pretzel link $P\left(p_{i} ; n\right)$ with $n_{+} P\left(p_{i} ; n\right)=1$ or $n_{-} P\left(p_{i} ; n\right)=1$, we know that 
$\alpha\left(P\left(p_{i} ; n\right)\right) \leqslant c\left(P\left(p_{i} ; n\right)\right)$. But we don't know yet whether $\alpha\left(P\left(p_{i} ; n\right)\right)=c\left(P\left(p_{i} ; n\right)\right)$ or not.

\section{Prime tANGles}

By a tangle we mean a 2-string tangle, which is a pair $(B, t)$ of a 3 -ball $B$ and two arcs $t$ properly embedded in $B$. A tangle without local knots is said to be locally trivial. Two tangles $(A, s)$ and $(B, t)$ are said to be equivalent if there is a homeomorphism from $(A, s)$ to $(B, t)$. If two arcs in a tangle $(B, t)$ can be separated by a 2 -disc properly embedded in a 3 -ball, the tangle $(B, t)$ is said to be separable. Otherwise, the tangle $(B, t)$ is said to be non-separable. A locally trivial separable tangle is said to be trivial. A regular diagram of a trivial tangle, or its spatial realisation, is said to be rational. A tangle is called a prime tangle if it is locally trivial and non-separable. A tangle sum of two tangles $(A, s)$ and $(B, t)$ is defined by the link $(A, s) \cup_{\pi}(B, t)$ obtained by gluing them together via a homeomorphism $\pi: \partial(B, t) \rightarrow \partial(A, s)$. The following proposition is well-known.

\section{Proposition $4.1[7,8,10]$}

(1) A link obtained from two prime tangles by any tangle sum is prime.

(2) Let $(C, v)$ be a tangle and $D$ a disc properly embedded in $C$ such that $D$ separates $(C, v)$ into two tangles $(A, s)$ and $(B, t)$ and that $(\partial A \backslash D) \cap v$, $(\partial B \backslash D) \cap v, D \cap v$ consist of two points. Suppose that the two tangles $(A, s)$ and $(B, t)$ are prime. Then $(C, v)$ is a prime tangle.

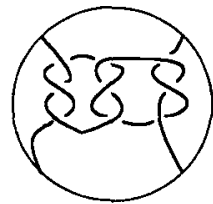

$\left(B_{1}, t_{1}\right)$

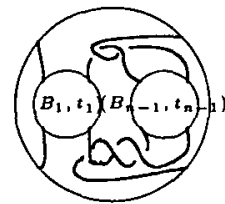

$\left(B_{n}, t_{n}\right)$

Figure 4.1

Let $\left(B_{1}, t_{1}\right), \ldots,\left(B_{n}, t_{n}\right)$ be the tangles defined inductively as in Figure 4.1 and let $P_{n}$ be the knot obtained from the tangle sum of two tangles $\left(B_{1}, t_{1}\right)$ and $\left(B_{n}, t_{n}\right)$ defined as in Figure 4.2. 


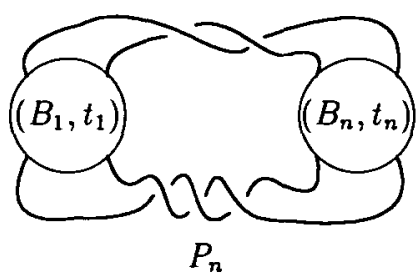

Figure 4.2

Propos ITION 4.2. For each positive integer $n$, we have

(1) The tangle $\left(B_{i}, t_{i}\right)$ is a prime tangle, for $1 \leqslant i \leqslant n$ and

(2) The knot $P_{n}$ is a prime knot.

Proof: (1) First, we claim that the tangle $\left(B_{1}, t_{1}\right)$ is prime. Since each of two arcs in the tangle $\left(B_{1}, t_{1}\right)$ is unknotted, the tangle $\left(B_{1}, t_{1}\right)$ is locally trivial. If it is separable, then it must be equivalent to the trivial tangle. Since a trivial tangle diagram induces a two bridge knot, the tangle $\left(B_{1}, t_{1}\right)$ induces a two bridge knot. But the tangle $\left(B_{1}, t_{1}\right)$ induces the pretzel knot $P(3,-3,3)$ which is not a two bridge knot. Thus the tangle $\left(B_{1}, t_{1}\right)$ is non-separable. Therefore it is prime. It follows from Proposition $4.1(2)$ that the tangles $\left(B_{i}, t_{i}\right), i=1, \ldots, n$, are prime tangles.

(2) Since the knot $P_{n}$ is a tangle sum of two tangles $\left(B_{1}, t_{1}\right)$ and $\left(B_{n}, t_{n}\right)$, this follows from Proposition 4.1 (1).

\section{ARC INDEXES AND ADEQUATE KNOTS}

We have seen that there is an adequate knot $K$ such that $\alpha(K)=c(K)$ in Theorem 3.3. In 1995, Cromwell [4] proved that $\alpha\left(L_{1} \# L_{2}\right)=\alpha\left(L_{1}\right)+\alpha\left(L_{2}\right)-2$. From this we have the following

Propos ITION 5.1. For each non-negative integer $n$, there is an adequate knot $K_{n}$ such that $\alpha\left(K_{n}\right)-c\left(K_{n}\right)=-2 n$.

Proof: Let $K_{0}$ be the pretzel knot $P(3,-3,3,-2)$ and let $K_{n}$ be the connected sum of $n+1$ copies of $K$. Then $\alpha\left(K_{0}\right)=c\left(K_{0}\right)$. Since a connected sum of adequate knots is again an adequate knot, $K_{n}$ is an adequate knot. Thus $c\left(K_{n}\right)=(n+1) c\left(K_{0}\right)$. Since $\alpha\left(L_{1} \# L_{2}\right)=\alpha\left(L_{1}\right)+\alpha\left(L_{2}\right)-2$ for any links $L_{1}$ and $L_{2}, \alpha\left(K_{n}\right)=(n+1) \alpha\left(K_{0}\right)-2 n=$ $c\left(K_{n}\right)-2 n$.

For each non-negative integer $n$, we are interested in finding an adequate and prime knot $K_{n}$ such that $\alpha\left(K_{n}\right)=c\left(K_{n}\right)-2 n$ and $\rho\left(K_{n}\right)=-n$. In the case $n=3$, we have the following example whose generalisation is Theorem 5.3. 
EXAMPLE 5.2. Let $D_{3}$ be the knot diagram of the knot $P_{3}$ introduced in Section 4 as depicted in Figure 5.1. Let $G$ be the associated graph of the knot diagram $D_{3}$ as in Figure 5.2. Since $G_{+}$and $G_{-}$have no isthmus and $\overline{G_{+}}$and $\overline{G_{-}}$have no loop, $D_{3}$ is an adeguate diagram. Thus $p_{0}\left(G_{+}\right)=1, p_{0}\left(G_{-}\right)=4$ and $\left|G_{+} \cap G_{-}\right|=8$. Hence $\rho\left(D_{3}\right)=-3$. Since $c\left(D_{3}\right)=51, \alpha\left(D_{3}\right) \geqslant c\left(D_{3}\right)+2 \rho\left(D_{3}\right)=51-6=45$. The knot diagram $D_{3}$ has a binding circle which intersects $D_{3}$ in 45 points as in Figure 5.3 and hence $\alpha\left(D_{3}\right)=45=c\left(D_{3}\right)-2 \cdot 3$.

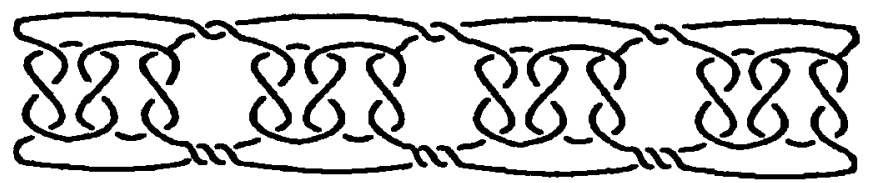

Figure 5.1

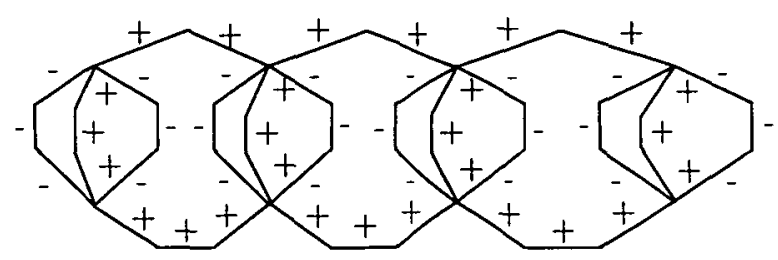

$D_{3}$

Figure 5.2

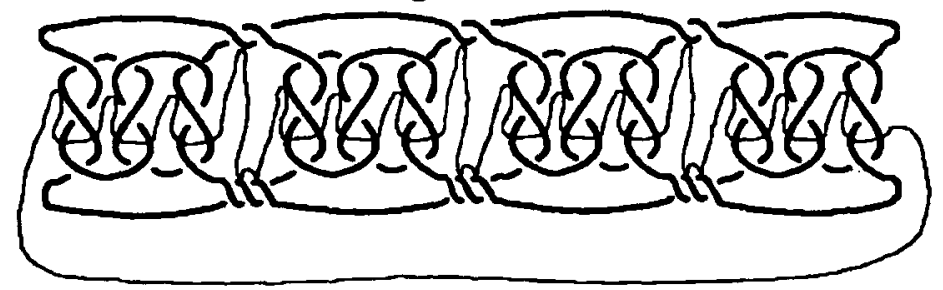

Figure 5.3

THEOREM 5.3. For each positive integer $n$, the prime knot $P_{n}$ is an adequate knot such that $\alpha\left(P_{n}\right)-c\left(P_{n}\right)=-2 n=2 \rho\left(P_{n}\right)$.

Proof: The proof proceeds in exactly the same way as in Example 5.2. Let $D_{n}$ be the knot diagram of the knot $P_{n}$ introduced in Section 4 and let $G$ be the associated graph of the knot diagram $D_{n}$. Since $G_{+}$and $G_{-}$have no isthmus and $\overline{G_{+}}$and $\overline{G_{-}}$ have no loop, $D_{n}$ is an adequate diagram. Then $p_{0}\left(G_{+}\right)=1, p_{0}\left(G_{-}\right)=n+1$ and $\left|G_{+} \cap G_{-}\right|=2 n+2$. Hence $\rho\left(P_{n}\right)=\rho\left(D_{n}\right)=1+(n+1)-(2 n+2)=-n$. Since $c\left(D_{n}\right)=9(n+1)+5 n=14 n+9$ and the knot diagram $D_{n}$ has a binding circle which intersects $D_{n}$ in $12 n+9$ points, $\alpha\left(D_{n}\right)=12 n+9=(14 n+9)+2(-n)=c\left(D_{n}\right)+2 \rho\left(D_{n}\right)$. Hence $\alpha\left(P_{n}\right)=c\left(P_{n}\right)-2 n$. 


\section{REFERENCES}

[1] Y. Bae and C.-Y. Park, 'An upper bound of arc index of links', Math. Proc. Cambridge Philos. Soc. (2000) (to appear).

[2] E. Beltrami and P.R. Cromwell, 'Minimal arc presentations of some non-alternating knots', Topology Appl. 81 (1997), 137-145.

[3] J.S. Birman and W.W. Menasco, 'Special positions for essential tori in link complements', Topology 33 (1994), 525-556.

[4] P.R. Cromwell, 'Embedding knots and links in an open book I : Basic properties', Topology Appl. 64 (1995), 37-58.

[5] P.R. Cromwell, 'Arc presentations of knots and links', in Proc. Knot Theory Conference Warsaw 1995, (V.F.R. Jones et al., Editors), Banach Center Publications (Polish Acad. Sci., Warsaw, 1998).

[6] P.R. Cromwell and I.J. Nutt, 'Embedding knots and links in an open book II : Bounds on arc index', Math. Proc. Cambridge Philos. Soc. 119 (1996), 309-319.

[7] A. Kawauchi, A Survey of knot theory (Birkhäuser Verlag, Basel, 1996).

[8] W.B.R. Lickorish, 'Prime knots and tangles', Trans. Amer. Math. Soc. 267 (1981), 321-332.

[9] H.R. Morton and E. Beltrami, 'Arc index and the Kauffman polynomial', Math. Proc. Cambridge Philos. Soc. 123 (1998), 41-48.

[10] Y. Nakanishi, 'Primeness of links', Math. Sem. Notes Kobe Univ. 9 (1981), 415-440.

[11] M.B. Thistlethwaite, 'Kauffman's polynomial and alternating links', Topology 27 (1988), 311-318.

[12] M.B. Thistlethwaite, 'On the Kauffman polynomial of an adequate link', Invent. Math. 93 (1988), 285-296.

Department of Mathematics

College of Natural Sciences

Kyungpook National University

Taegu 702-701

South Korea 